\title{
A Simple Baseline for Travel Time Estimation using Large-scale Trip Data
}

\author{
HONGJIAN WANG, Twitter Inc., USA \\ XIANFENG TANG, YU-HSUAN KUO, DANIEL KIFER, and ZHENHUI LI, \\ Pennsylvania State University, USA
}

\begin{abstract}
The increased availability of large-scale trajectory data provides rich information for the study of urban dynamics. For example, New York City Taxi \& Limousine Commission regularly releases source/destination information of taxi trips, where 173 million taxi trips released for Year 2013 [29]. Such a big dataset provides us potential new perspectives to address the traditional traffic problems. In this article, we study the travel time estimation problem. Instead of following the traditional route-based travel time estimation, we propose to simply use a large amount of taxi trips without using the intermediate trajectory points to estimate the travel time between source and destination. Our experiments show very promising results. The proposed big-datadriven approach significantly outperforms both state-of-the-art route-based method and online map services. Our study indicates that novel simple approaches could be empowered by big data and these approaches could serve as new baselines for some traditional computational problems.
\end{abstract}

CCS Concepts: • Applied computing $\rightarrow$ Transportation; • Information systems $\rightarrow$ Nearest-neighbor search; $\bullet$ Computing methodologies $\rightarrow$ Unsupervised learning;

Additional Key Words and Phrases: Travel time estimation, big data, baseline, trajectory data

\section{ACM Reference format:}

Hongjian Wang, Xianfeng Tang, Yu-Hsuan Kuo, Daniel Kifer, and Zhenhui Li. 2019. A Simple Baseline for Travel Time Estimation using Large-scale Trip Data. ACM Trans. Intell. Syst. Technol. 10, 2, Article 19 (January 2019), 22 pages.

https://oi.org/10.1145/3293317

\section{INTRODUCTION}

Positioning technology is widely adopted into our daily life. On-board GPS devices track the operation of vehicles and provide navigation service; meanwhile, a significant amount of trajectory

The work was supported in part by NSF Awards No. 1054389, No. 1228669, No. 1544455, No. 1652525, No. 1618448, and No. 1702760. Zhenhui Li acknowledges the support from Haile Family Early Career Professorship. The views and conclusions contained in this article are those of the authors and should not be interpreted as representing any funding agencies.

Authors' addresses: H. Wang, Twitter Inc. 1355 Market St. \#900, San Francisco, CA, 94103, USA; email: hongjianw@ twitter.com; X. Tang and Y.-H. Kuo, Pennsylvania State University, Westgate Building, University Park, PA, 16802, USA, emails: xut10@ist.psu.edu, yzk5145@cse.psu.edu; D. Kifer, Pennsylvania State University, W333 Westgate Building, University Park, PA, 16802, USA; email: dkifer@cse.psu.edu; Z. Li, Pennsylvania State University, E331 Westgate Building, University Park, PA, 16802, USA; email: jessieli@ist.psu.edu.

The work was done while H. Wang was a PhD candidate at Penn State University.

Permission to make digital or hard copies of all or part of this work for personal or classroom use is granted without fee provided that copies are not made or distributed for profit or commercial advantage and that copies bear this notice and the full citation on the first page. Copyrights for components of this work owned by others than ACM must be honored. Abstracting with credit is permitted. To copy otherwise, or republish, to post on servers or to redistribute to lists, requires prior specific permission and/or a fee. Request permissions from permissions@acm.org.

(C) 2019 Association for Computing Machinery.

2157-6904/2019/01-ART19 \$15.00

https://doi.org/10.1145/3293317 


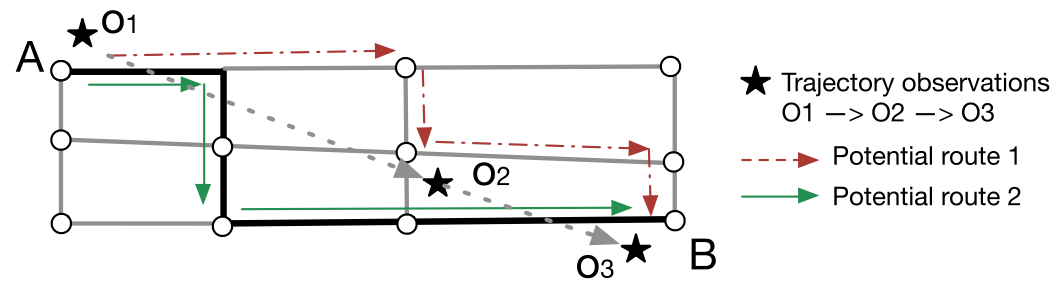

Fig. 1. Use historical trajectory $o_{1} \rightarrow o_{2} \rightarrow o_{3}$ to estimate the travel time from $A$ to $B$.

data are collected. For example, a huge amount of taxi trip data is accumulated and enables us to study urban dynamics. While many existing methods try to outdo each other in terms of complexity and algorithmic sophistication, in the spirit of "big data beats algorithms," we study whether some simple baseline methods can be empowered by taking the benefit of big data.

In this article, we revisit a traditional travel time estimation problem, which estimates the travel time between an origin and a destination. Existing travel time estimation approaches mostly fall into the line of route-based method [4, 5, 13, 31]. Given a source and a destination, these methods first identify a route and then estimate the travel time for this route by aggregating the travel time spent on each segment (or subpath) based on historical trajectories. Such a route-based method faces many complicated challenges in implementation. In particular, there are two major challenges:

- Route mapping. The route-based method needs to obtain the travel time from historical trajectories. But the actual trajectory data may not map well onto the roads due to GPS positioning errors, imprecise road network data, or the time gap between two adjacent observations (for example, in the Shanghai taxi data used in our experiments, the GPS sample interval ranges from 60 to 90s). We illustrate this problem in Figure 1. Suppose we are estimating the travel time from A to B. We need to first identify a route (the black road segments). Next, to estimate the travel time for this route, we need to know the travel time spent on each segment. And the time spent on each segment is obtained from historical trajectories. One historical trajectory $o_{1} \rightarrow o_{2} \rightarrow o_{3}$ is shown in Figure 1. However, it is non-trivial to map these GPS points to the actual road segments. Two potential routes are plotted in Figure 1 and both could be taken by the observed trajectory. In addition, we often have missing data between two consecutive data points. Therefore, it is not clear what is the actual route taken by an observed trajectory.

- Data sparsity. Trajectory data are sparse. Even with millions of trip observations, there are a lot of road segments not covered by any trajectory because of the non-uniform spatial distribution of GPS locations in the city. For example, in the Shanghai taxi data, even with over 300 million GPS records, more than $50 \%$ of road segments are not covered by any trajectory. The inference of travel time is not accurate due to the limited number of trajectories covering the road segment. In addition to the spatial sparseness, the trajectory data are temporally sparse too. Even if a road segment is covered by a few trajectories, these trajectories may not be sufficient to estimate the dynamic travel time that varies under different conditions (e.g., peak time and weekends).

To avoid these challenges in the route-based method, we propose a simple baseline method to estimate the travel time. Suppose we are to predict travel time from $A$ to $B$ and we have hundreds of historical trips from $A$ to $B$. Even if we do not have the intermediate points for those trips, 
the average travel time of these historical trips could provide us a reasonable estimation of the travel time from $A$ to $B$. This is similar to the way we humans tackle this problem. For example, if someone asks you how long it takes to drive from your office to home, you do not calculate the time based on the travel time on each road segments. Instead, you would estimate an approximate time based on the historical travel times.

But in the real-world setting, even with a large database of millions of trips, it is not easy to get a sufficient number of trips from exactly the same origin $A$ to exactly the same destination $B$. Therefore, we look for neighboring trips with a nearby origin and destination to estimate the travel time and we call our method a neighbor-based method. However, simply taking the average time of neighboring trips will yield a poor performance because of the variance in traffic at different times. For example, the travel time during the peak hour could be much longer than that at midnight; there could also be abnormal traffic changes due to holidays or traffic jams. Therefore, we further propose to consider such traffic dynamics by exploiting traffic periodicity and making timely adjustments based on recent traffic patterns.

One may argue that our neighbor-based method does not provide the specific route information, which limits its application. While this is true, it does not keep our method from being a good baseline for travel time estimation problem. Besides, there are many real scenarios where the route information is not important. One example is trip planning [14]. For example, a person will take a taxi to catch a flight, which leaves at 5 p.m. Since he is not driving, the specific route is less of a concern and he only needs to know the trip time. The second example is to estimate the city commuting efficiency $[17,19]$. In the field of urban transportation research, the commuting efficiency measure is a function of observed commuting time and expected minimum commuting time of given origin and destination areas. To estimate the commuting efficiency of a city for a future time, the ability to predict the pairwise travel time for any two areas is crucial. In this scenario, the specific path between two areas is not important, either.

We conduct experiments on two large real-world datasets. We evaluate our method using more than 450 million NYC trips, and our method is able to outperform Bing Maps [16] by almost 30\% (even when Bing uses traffic conditions during the same relative time within a week as query trips). Since NYC taxi data only contain the information on endpoints of the trips (i.e., pick-up and drop-off locations), to compare our method with the route-based method, we use Shanghai taxi data with more than 5 million trips with complete intermediate points of trajectories. On this dataset, our method also significantly outperforms the state-of-the-art route-based method [27] by $19 \%$ and Baidu Maps by $17 \%$. It is noteworthy that our method runs 40 times faster than the state-of-the-art route-based method.

In summary, the contributions of this article are as follows:

- We propose to estimate travel time using neighboring trips from the large-scale historical data. To the best of our knowledge, this is the first work to estimate travel time without computing the routes.

- We improve our neighbor-based approach by addressing the dynamics of traffic conditions.

- Our experiments are conducted on large-scale real data. We show that our method can outperform state-of-the-art methods as well as online map services (Bing Maps and Baidu Maps).

The rest of the article is organized as follows. Section 2 reviews related work. Section 3 defines the problem and provides an overview of the proposed approach. Section 4 discusses our method to weight the neighboring trips. We present our experimental results in Section 5 and conclude the paper in Section 6. 


\section{RELATED WORK}

To the best of our knowledge, most of the studies in literature focus on the problem of estimating travel time for a route (i.e., a sequence of locations). There are two types of approaches for this problem: segment-based method and path-based method.

Segment-based method. A straightforward travel time estimation approach is to estimate the travel time on individual road segments first and then take the sum over all the road segments of the query route as the travel time estimation. There are two types of data that are used to estimate the travel time on road segments: loop detector data and floating-car data (or probe data) [23]. Loop detector can sense whether a vehicle is passing above the sensor. Various methods [12, 20, 22, 26] have been proposed to infer vehicle speed from the loop sensor readings and then infer travel time on individual road segments.

Floating cars collect timestamped GPS coordinates via GPS receivers on the cars. The speed of individual road segments at a time $t$ can be inferred if a floating car is passing through the road segment at that time point $[2,30]$. Due to the low GPS sampling rate, a vehicle typically goes through multiple road segments between two consecutive GPS samplings. A few methods have been proposed to overcome the low sampling rate issue [3, 8, 9, 11,28]. Another issue with floatingcar data is data sparsity-not all road segments are covered by vehicles all the time. Wang et al. [27] proposes to use matrix factorization to estimate the missing values on individual road segments. In our problem setting, we assume the input is a special type of the floating-car data, where we only know the origin and destination points of the trips.

Path-based method. Segment-based method does not consider the transition time between road segments such as waiting for traffic lights and making left/right turns. Recent research works start considering the time spent on intersections $[6-8,15]$. However, these methods do not directly use sub-paths to estimate travel time. Rahmani et al. [21] and Wang et al. [27] propose to concatenate sub-paths to give a more accurate estimation of the query route. Our proposed solution can be considered as an extreme case of the path-based method, where we use the travel time of the full paths in historical data to estimate the travel time between an origin and a destination. Full paths are better at capturing the time spent on all intersections along the routes, assuming we have enough full paths (i.e., a reasonable support).

Origin-destination travel time estimation. In our problem setting, instead of giving a route as the input query, we only take the origin and destination as the input query. One could argue that we could turn the problem into the trajectory travel time estimation by first finding a route (e.g., shortest route or fastest route) $[4,5,13,31]$ and then estimating the travel time for that route. This alternative solution is similar to those provided by online maps services, where users input a starting point and an ending point, and the service generates a few route options and their corresponding times. Such a solution eventually leads to travel time estimation of a query route. However, the historical trajectory data may only have the starting and ending points of the trips, such as the public NYC taxi data [29] used in our experiment. Different from the data used in literature, which has the complete trajectories of floating cars, such data have limited information and are not suitable for the segment-based method or path-based method discussed above. In addition, route-based approach introduces more expensive computations, because we need to find the route first and then compute travel time on segments or sub-paths. More importantly, such expensive computation does not necessarily lead to better performance compared with our method using limited information, as we will demonstrate in the experiment section. 


\section{PROBLEM DEFINITION}

A trip $\mathrm{p}_{i}$ is defined as a 5-tuple $\left(o_{i}, d_{i}, s_{i}, l_{i}, t_{i}\right)$, which consists of the origin location $o_{i}$, the destination location $d_{i}$ and the starting time $s_{i}$. Both origin and destination locations are GPS coordinates. We further use $l_{i}$ and $t_{i}$ to denote the distance and travel time for this trip, respectively. Note that, here we assume the intermediate locations of the trips are not available. In real world applications, it is quite possible that we can only obtain such limited information about trips due to privacy concerns and tracking costs. For example, the largest public taxi data released by New York City [29] does not contain any intermediate GPS points.

Problem 1 (OD Travel Time Estimation). Suppose we have a database of trips, $\mathcal{D}=\left\{\mathbf{p}_{i}\right\}_{i=1}^{N}$. Given a query $\mathrm{q}=\left(o_{q}, d_{q}, s_{q}\right)$, our goal is to estimate the travel time $t_{q}$ with given origin $o_{q}$, destination $d_{q}$, and departure time $s_{q}$, using the historical trips in $\mathcal{D}$.

\subsection{Approach Overview}

An intuitive solution is that we should find similar trips as the query trip q and use the travel time of those similar trips to estimate the travel time for q. The problem can be decomposed to two sub-problems: (1) how to define similar trips; and (2) how to aggregate the travel time of similar trips. Here, we name similar trips as neighboring trips (or simply neighbors) of query trip q. The naive solution to travel time estimation is to find neighboring trips with exact origin/destination and trip starting time. However, even with millions of trips due to the distribution skewness, this naive solution faces with serious sparsity issue. Therefore, we have to loose the condition for neighboring trips by considering trips with varying starting times, and thus the aggregating is not trivial because of varying traffic conditions.

We call trip $\mathbf{p}_{i}$ a neighbor of trip $\mathbf{q}$ if the origin (and destination) of $\mathbf{p}_{i}$ are spatially close to the origin (and destination) of $\mathbf{q}$. Thus, the set of neighbors of $\mathbf{q}$ is defined as

$$
\mathcal{N}(\mathbf{q})=\left\{\mathbf{p}_{i} \in \mathcal{D} \mid \operatorname{dist}\left(o_{i}, o_{q}\right) \leq \tau \text { and } \operatorname{dist}\left(d_{i}, d_{q}\right) \leq \tau\right\},
$$

where $\operatorname{dist}()$ is a distance measure of two given points. For simplicity, we use the Euclidean distance in our article, but it is better to use the road network distance.

With the definition of neighbors, a baseline approach is to take the average travel time of these trips as the estimation:

$$
\widehat{t}_{q}=\frac{1}{|\mathcal{N}(\mathbf{q})|} \sum_{\mathbf{p}_{i} \in \mathcal{N}(\mathbf{q})} t_{i} .
$$

An alternative definition of neighbors is to weight neighboring trips based on the origin distance (i.e., $\left.\operatorname{dist}\left(o_{i}, o_{q}\right)\right)$ and destination distance (i.e., $\left.\operatorname{dist}\left(d_{i}, d_{q}\right)\right)$. However, such a definition will make the computation more expensive, since we typically need to consider more neighbors and also does not lead to a better performance (refer to Section 5.3.4).

With the model in Equation (2), there is a major issue with the baseline approach: it does not model the dynamic traffic conditions across different time. To remedy this issue, for each neighboring trip $\mathrm{p}_{i}$ of $\mathbf{q}$, we define the scaling factor $w_{i}$ calculated from the speed reference, so that $w_{i} t_{i} \approx t_{q}$. Thus, our estimation model becomes

$$
\widehat{t}_{q}=\frac{1}{|\mathcal{N}(\mathbf{q})|} \sum_{\mathrm{p}_{i} \in \mathcal{N}(\mathrm{q})} w_{i} t_{i} .
$$

In the following sections, we will discuss how to obtain scaling factor $w_{i}$ by using the temporal regularity (Section 4.1), adjusting with respect to irregular changes (Section 4.2), and further considering the geographical differences (Section 4.3). 


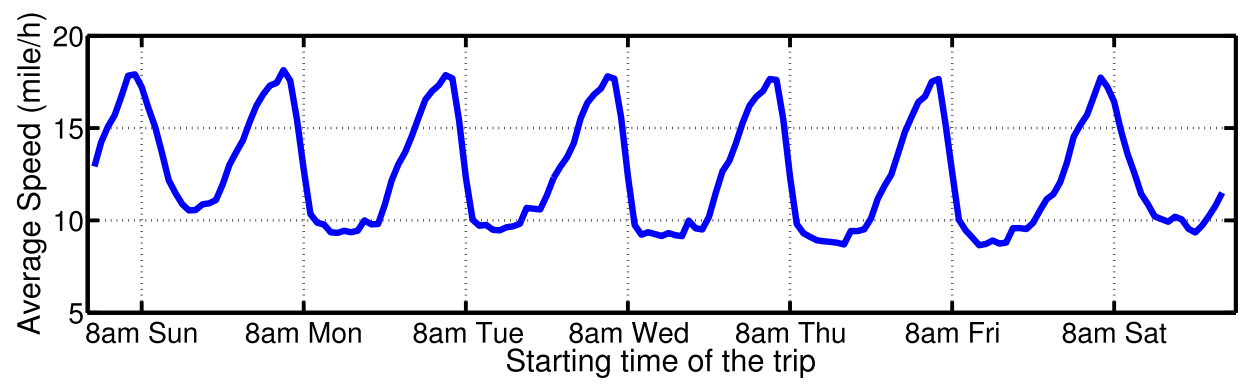

Fig. 2. Average travel speed w.r.t. trip starting time.

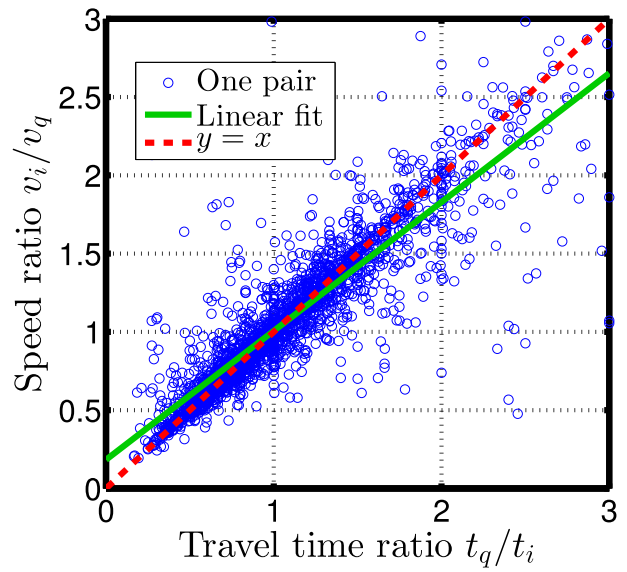

(a) $\frac{t_{q}}{t_{i}} \approx \frac{v_{i}}{v_{q}}$

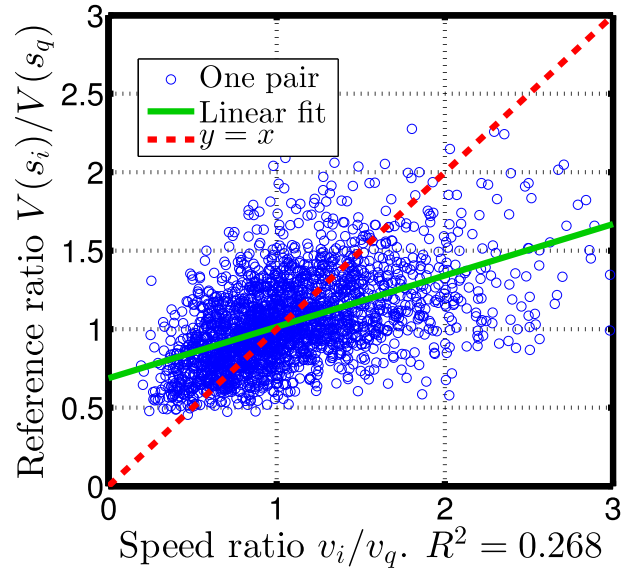

(b) $\frac{v_{i}}{v_{q}} \approx \frac{V\left(s_{i}\right)}{V\left(s_{q}\right)}$

Fig. 3. The validity of our assumptions. We randomly sample a set of neighboring trip pairs, and calculate the different ratios for each pair. We plot each trip pair as a blue point. The solid green line is the linear regression fit of all the points. The dotted red line is $y=x$. In (a), we can see that fitted line has a slope approximating 1 , which verifies our assumption $\forall \mathrm{p}_{i} \in \mathcal{N}(q), l_{i} \simeq l_{q}$. Similarly, in (b), we conclude that ratio of speed reference approximates the ratio of actual speed.

\section{CAPTURING THE TEMPORAL DYNAMICS OF TRAFFIC CONDITIONS}

As we discussed earlier, it is not appropriate to simply take the average of all the neighbors of $\mathbf{q}$ because of traffic conditions vary at different times. Figure 2 shows the average speed of all NYC taxi trips at different times in a week. Apparently, the average speed is much faster at the midnight compared with the speed during the peak hours. Thus, if we wish to estimate the travel time of a trip $\mathbf{q}$ at 2 a.m. using a neighboring trip $\mathbf{p}$ at 5 p.m., we should proportionally decrease the travel time of $\mathbf{p}$, because a 2 a.m. trip is often much faster than a 5 p.m. trip.

Now the question is, how can we derive a temporal scaling reference to correspondingly adjust travel time on the neighboring trips. We first define the scaling factor of a neighboring trip $\mathbf{p}_{i}$ on query trip q as

$$
w_{i}=\frac{t_{q}}{t_{i}} .
$$

One way to estimate $w_{i}$ is using the speed of $\mathbf{p}_{i}$ and $\mathbf{q}$. Let $v_{i}$ and $v_{q}$ be the speed of trip $\mathbf{p}_{i}$ and q. Since we pick a small $\tau$ to extract neighboring trips of $\mathbf{q}$, it is safe to assume that $\forall \mathrm{p}_{i} \in \mathcal{N}(q)$, $l_{i} \simeq l_{\mathrm{q}}$. In Figure 3(a), on a sample set of neighboring trip pairs, we plot the inverse speed ratio 


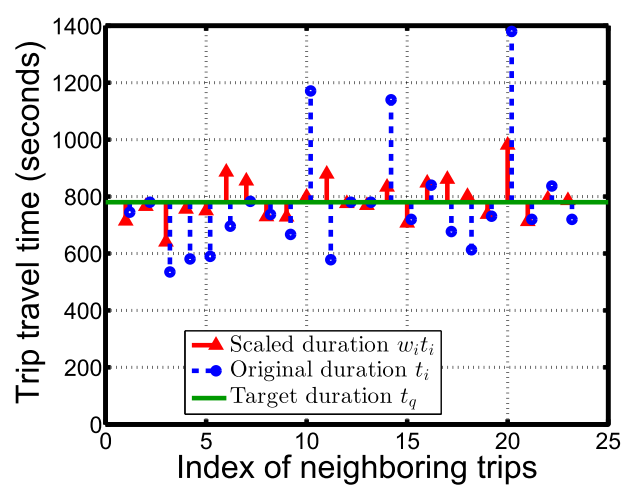

(a)

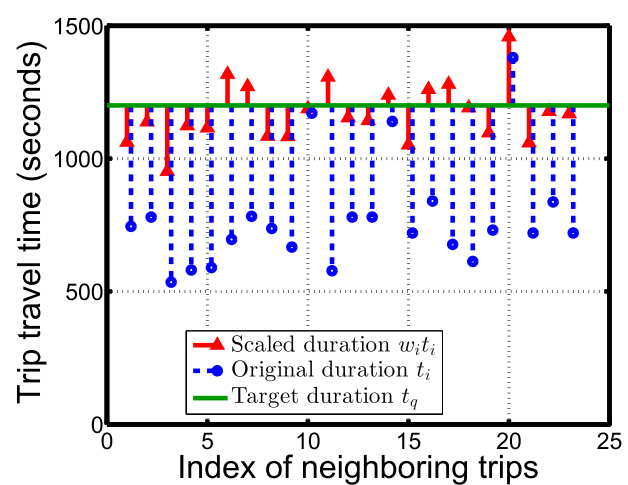

(b)

Fig. 4. We pick two particular trips to demonstrate the effectiveness of the scaling factor. The horizontal green line shows the travel time of target trip q. The actual travel time $t_{i}$ of neighboring trips is plotted as blue circle, while the scaled travel time $w_{i} t_{i}$ is plotted as red triangle. The index of neighboring trips refers to the ID of neighboring trip. In (a), the actual travel times of historical trips have very large variance, because they are from different time slots with distinct traffic conditions. The scaling factor successfully reduces the variance in the actual travel time $t_{i}$. In (b), the target trip q occurred in rush hour, and thus $t_{q}$ is much longer than most of its neighboring trips. The scaling factor successfully fill in the gap between historical trips and $\mathbf{q}$.

against the travel time ratio. The solid line shows the actual relation between $\frac{t_{q}}{t_{i}}$ and $\frac{v_{i}}{v_{q}}$, which is very close to the dotted line $y=x$, which means that two ratios are approximately equivalent. With this assumption, we have

$$
w_{i}=\frac{t_{q}}{t_{i}}=\frac{l_{q} / v_{q}}{l_{i} / v_{i}} \approx \frac{v_{i}}{v_{q}} .
$$

However, $v_{q}$ is unknown, so we need to estimate $\frac{v_{i}}{v_{q}}$. Since the average speed of all trips are stable and readily available, we try to build a bridge from the actual speed ratio to the corresponding average speed ratio for any given two trips. One solution is to assume the ratio between $v_{q}$ and $v_{i}$ approximately equals to the ratio between the average speed of all trips at $s_{q}$ and $s_{i}$. Formally, let $V(s)$ denote the average speed of all trips at timestamp $s$, we have an approximation of $w_{i}$ as

$$
w_{i} \approx \frac{v_{i}}{v_{q}} \approx \frac{V\left(s_{i}\right)}{V\left(s_{q}\right)} .
$$

This assumption is validated in Figure 3(b). For the sample set of neighboring trip pairs, the average speed ratio is plotted against actual speed ratio. Since the points are approximately distributed along the line $y=x$, we conclude that average speed ratio is a feasible approximation of the scaling factor. We notice that the fitted line has a slope less than 1 , which is mainly due to the anomaly in individual trips. Specifically, the speed of individual trips $v$ has a higher variance and some individual trip pairs have an extreme large ratio. However, the average speed $V(s)$ has a smaller variance and the ratio is confined to a more reasonable range $[0.5,2]$.

Next, we show the effectiveness of the scaling factor calculated from Equation (5). In Figure 4, we present two specific trips to demonstrate the intuition of how the scaling factor works. For each trip, we retrieve its historical neighboring trips, and then plot the actual travel time in circle together with the scaled travel time in triangle. From Figure 4(a), we can see that the scaling factor helps reduce the variance in the travel time of neighboring trips. In Figure 4(b), trip q happened 


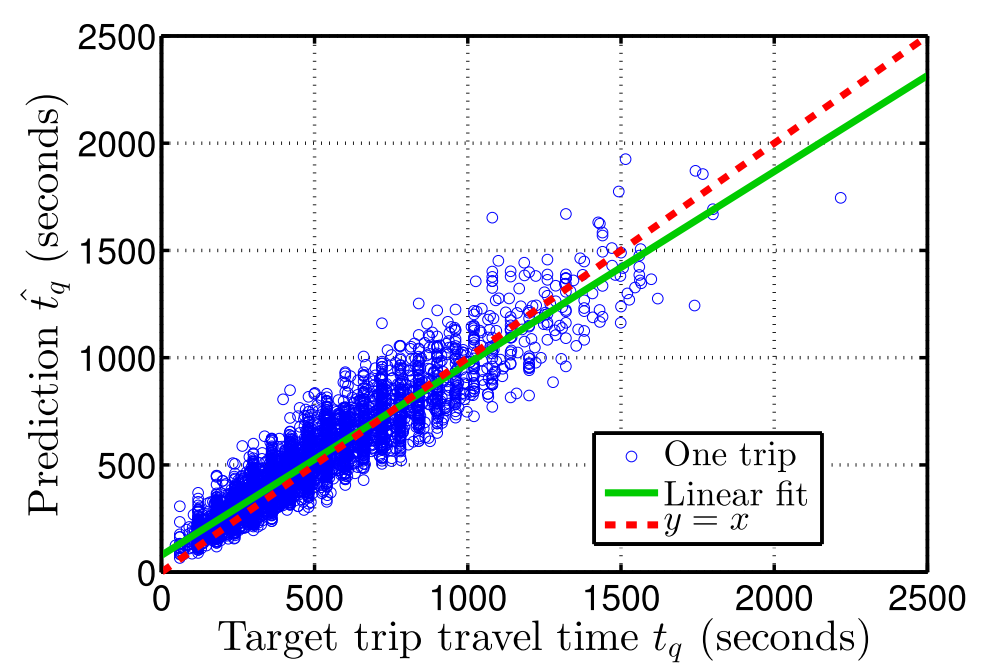

Fig. 5. Estimated travel time against actual travel time. Each point is a trip, where the estimation $\hat{t_{q}}$ is plotted against $t_{q}$. The green line is the linear regression fit of the points, which is closer to the red line $y=x$. This means the prediction is close to the actual value.

in rush hour, which takes longer time than most of its neighboring trips. This gap is successfully filled in by the scaling factor.

Considering such scaling factors using speed as the reference, we can estimate the travel time of $\mathrm{q}$ using the neighboring trips as follows:

$$
\widehat{t}_{q}=\frac{1}{|\mathcal{N}(\mathbf{q})|} \sum_{\mathbf{p}_{i} \in \mathcal{N}(\mathbf{q})} t_{i} \cdot \frac{V\left(s_{i}\right)}{V\left(s_{q}\right)} .
$$

We show the effectiveness of this predictor in Figure 5. Each point in the figure is a target trip. The prediction is plotted against the actual trip travel time. We can see that the prediction is close to the actual value. This indirectly implies the validity of assumptions we made previously.

To compute the average speed $V\left(s_{i}\right)$, we need to collect all the trips in $\mathcal{D}$, which start at time $s_{i}$. However, for the query trip q, the starting time $s_{q}$ may be the current time or some time in the future. Therefore, no trips in $\mathcal{D}$ have the same starting time as q. In the following, we discuss two approaches to predict $V\left(s_{q}\right)$ using the available data.

\subsection{Relative Speed Reference}

In this section, we assume that $V(s)$ exhibits a regular daily or weekly pattern. We fold the time into a relative time window $\mathcal{T}_{\text {rela }}=\{1,2, \ldots, T\}$, where $T$ is the assumed periodicity. For example, using a weekly pattern with 1 hour as the basic unit, we have $T=7 \times 24=168$. Using this relative time window, we represent the average speed of the $k$ th time slot as $V_{k}, \forall k \in \mathcal{T}_{\text {rela }}$. We call $\left\{V_{k} \mid k \in \mathcal{T}_{\text {rela }}\right\}$ relative reference.

We use $k_{i}$ to denote the time slot to which $s_{i}$ belongs. As a result, we can write $V\left(s_{i}\right)=V_{k_{i}}$. To compute $V_{k_{i}}$, we collect all the trips in $\mathcal{D}$, which fall into the same time slot as $\mathbf{p}_{i}$ and denote the set as $S\left(\mathrm{p}_{i}\right)$. Then, we have

$$
V_{k_{i}}=\frac{1}{\left|S\left(\mathbf{p}_{i}\right)\right|} \sum_{\mathbf{p}_{j} \in S\left(\mathbf{p}_{i}\right)} \frac{l_{j}}{t_{j}} .
$$




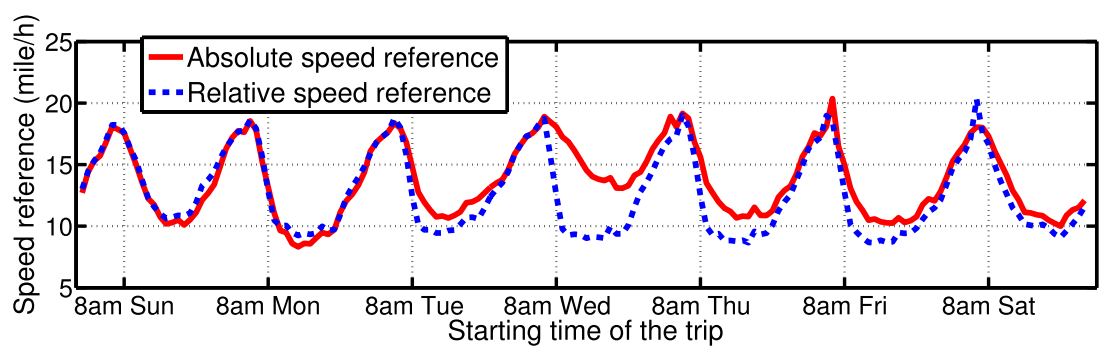

Fig. 6. Comparison between the absolute speed reference and the relative speed reference in the Christmas week (December 22, 2015-December 28, 2013). We can see the traffic condition is much better during Wednesday daytime than usual, because people are celebrating Christmas.

In Figure 2, we present the weekly relative speed reference on all trips. We can see all the weekdays share similar patterns the rush hour started from 8:00 a.m. Meanwhile, during the weekends, the traffic is not as much as usual during 8:00 a.m.

The relative speed reference mainly has the following two advantages. First, the relative speed reference is able to alleviate the data sparsity issue. By folding the data into a relative window, we will have more trips to estimate an average speed with a higher confidence. Second, the computation overhead of relative speed reference is small, and we could do it offline.

\subsection{Absolute Speed Reference}

In the previous section, the relative speed reference assumes the speed follows daily or weekly regularity. However, in real scenario, there are always irregularities in the traffic condition. For example, during national holidays the traffic condition will significantly deviate from the usual days. In Figure 6, we show the actual average traffic speed during the Christmas week (December 22, 2013-December 28, 2013). Compared with the relative speed reference, we can see that on December 25, 2013, the traffic condition is better than usual during the day, since people are celebrating Christmas. Therefore, assuming we have enough data, it would be more accurate if we could directly infer the average speed at any time slot $t$ from the historical data.

In this section, we propose an alternative approach to directly capture the traffic condition at different time slots. We extend our relative reference from Section 4.1 to an absolute speed reference. To this end, we partition the original timeline into time slots based on a certain time interval (i.e., 1h). All historical trips are mapped to the absolute time slots $\mathcal{T}_{a b s}=\{1,2,3, \cdots\}$ accordingly, and the average speed $\left\{V_{k} \mid k \in \mathcal{T}_{a b s}\right\}$ are calculated as the absolute speed reference.

The challenge in absolute speed reference is that for a given query trip with starting time $s_{q}$ in the near future (e.g., next hour), we need to estimate the speed reference $V\left(s_{q}\right)$. We estimate $V\left(s_{q}\right)$ by taking into account factors such as the average speed of previous hours, seasonality, and random noise. Formally, given the time series of average speed: $\left\{V_{1}, V_{2}, \ldots, V_{M}\right\}$, our goal is to compute $V_{M+1}$ as follows:

$$
V_{M+1}=f\left(V_{1}, \ldots, V_{M}\right)
$$

To predict current speed reference based on historical speed time series, we adopt the autoregressive integrated moving average (ARIMA) model [10] for time series forecasting. We will discuss the ARIMA model and seasonal ARIMA model in the following section.

4.2.1 Overview of the ARIMA Model. In statistical analysis of time series, ARIMA is a popular tool for understanding and predicting future values in a time series. Mathematically, for any time 
series $\left\{X_{t}\right\}$, let $L$ be the lag operator:

$$
L X_{t}=X_{t-1}, \forall t>1 .
$$

Then, the $\operatorname{ARIMA}(p, d, q)$ model is given by

$$
\left(1-\sum_{i=1}^{p} \phi_{i} L^{i}\right)(1-L)^{d} X_{t}=\left(1+\sum_{i=1}^{q} \theta_{i} L^{i}\right) \epsilon_{t},
$$

where parameters $p, d$, and $q$ are non-negative integers that refer to the order of the autoregressive, integrated, and moving average parts of the model, respectively. In addition, $\epsilon_{t}$ is a white noise process.

In practice, the autocorrelation function $(\mathrm{ACF})$ and partial autocorrelation function (APCF) is frequently used to estimate the order parameters $(p, d, q)$ from a time series of observations $\left\{X_{1}, X_{2}, \ldots, X_{M}\right\}$. Then, the coefficients $\left\{\phi_{i}\right\}_{i=1}^{p}$ and $\left\{\theta_{i}\right\}_{i=1}^{q}$ of the $\operatorname{ARIMA}(p, d, q)$ can be learned using standard statistical methods such as the least squares.

4.2.2 Incorporating the Seasonality. In our problem, the average speed $V_{t}$ exhibits a strong weekly pattern. Thus, instead of directly applying the ARIMA model to $\left\{V_{t}\right\}$, we first compute the sequence of seasonal difference $\left\{Y_{t}\right\}$ :

$$
Y_{t}=V_{t}-V_{t-T}
$$

where $T$ is the period (e.g., one week). Then, we apply the ARIMA model to $\left\{Y_{t}\right\}$ :

$$
\left(1-\sum_{i=1}^{p} \phi_{i} L^{i}\right)(1-L)^{d} Y_{t}=\left(1+\sum_{i=1}^{q} \theta_{i} L^{i}\right) \epsilon_{t} .
$$

Note that our ARIMA model with the seasonal difference is a special case of the more general class of Seasonal ARIMA (SARIMA) model for time series analysis. We refer interested readers to Reference [10] for detailed discussion about the model.

Suppose we use first-order difference of $Y_{t}$, namely, $d=1$ and $(1-L)^{d} Y_{t}=Y_{t}-Y_{t-1}$. Then, we have

$$
Y_{t}=Y_{t-1}+\sum_{i=1}^{p} \phi_{i} L^{i}\left(Y_{t}-Y_{t-1}\right)+\sum_{i=1}^{q} \theta_{i} L^{i} \epsilon_{t}+\epsilon_{t}
$$

Since the last term $\epsilon_{t}$ in Equation (13) is white noise, whose value is unknown but the expectation $E\left(\epsilon_{t}\right)=0$, we have estimator $\hat{Y}_{t}=Y_{t}-\epsilon_{t}$. Together with Equation (11), we have

$$
\hat{V}_{t}=\hat{Y}_{t}+V_{t-T} \text {. }
$$

\subsection{The Effect of Geographic Regions}

So far, we have assumed that the traffic condition follows the same temporal pattern across all geographic locations. However, in practice, trips within a large geographic area (e.g., New York City) may have different traffic patterns, depending on the spatial locations. For example, in Figure 7 we show the speed references of two different pairs of regions. The speed reference in region pairs $(A, B)$ has a larger variation than $(C, D)$. In particular, for $(A, B)$ pair, the average speed is $22.7 \mathrm{mph}$ at 4:00 a.m. on Thursday and $8.5 \mathrm{mph}$ at 12:00 p.m. on Wednesday. But for $(C, D)$ pair, the average speed is only 11.2 and $7.0 \mathrm{mph}$ at these two corresponding times. The reason could be that $A$ is a residential area, whereas $B$ is a business district. Therefore, the traffic pattern between $A$ and $B$ exhibits a very strong daily peak-hour pattern. However, regions $C$ and $D$ are popular tourist areas, the speeds are constantly slower compared with that of $(A, B)$. 


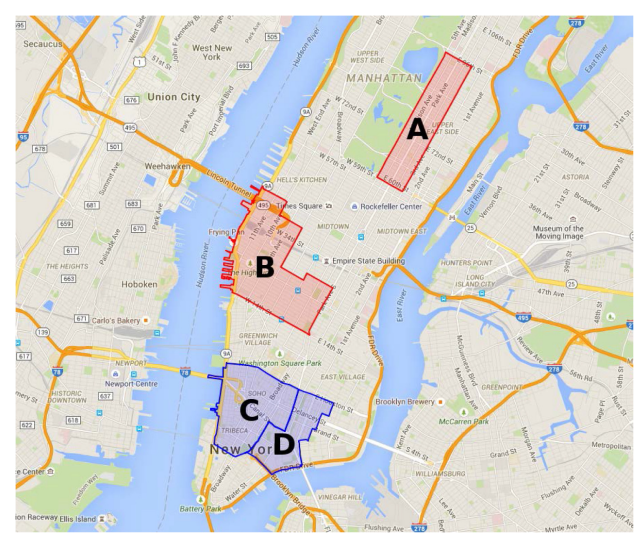

(a) Manhattan

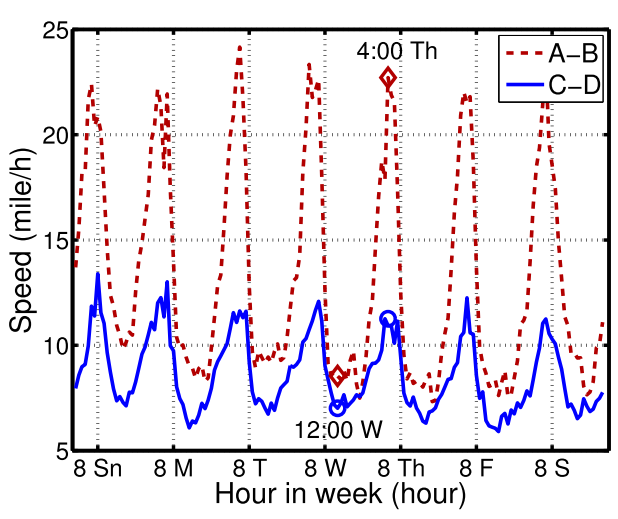

(b) Speed reference

Fig. 7. Different region pairs have different traffic patterns.

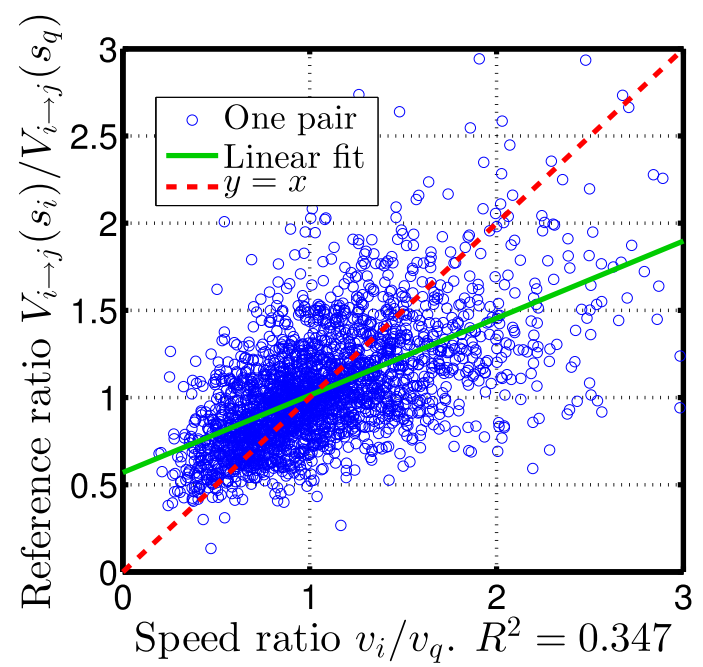

Fig. 8. Refining speed reference with regions improves the performance. We use the same set of trip pairs sampled in Figure 3(b) to validate $\frac{V_{i \rightarrow j}\left(s_{i}\right)}{V_{i \rightarrow j}\left(s_{q}\right)} \approx \frac{v_{i}}{v_{q}}$. Compared with Figure 3(b), the slope of linear regression fit is closer to 1 and the $R^{2}$ is larger, which means the region-based speed reference approximates the actual speed ratio better.

The real case above suggests that refining the speed references for different spatial regions could help us further improve the travel time estimation. Therefore, we propose to divide the map into a set of $K$ neighborhoods $\left\{R_{1}, R_{2}, \ldots, R_{K}\right\}$. For each pair of neighborhoods $\left(R_{i}, R_{j}\right), 1 \leq i, j \leq K$, we use $V_{i \rightarrow j}(s)$ to denote the average speed of all trips from $R_{i}$ to $R_{j}$ at starting time $s$. Let $\mathcal{D}_{i \rightarrow j}$ be the subset of trips in $\mathcal{D}$ whose origin and destination fall in $R_{i}$ and $R_{j}$, respectively. Then, $V_{i \rightarrow j}(s)$ can be estimated using $\mathcal{D}_{i \rightarrow j}$ in the same way as described in Sections 4.1 and 4.2.

We use Figure 8 to show the effectiveness of refining the speed reference for each region pairs. In Figure 8, we plot the refined speed reference ratio against the actual speed ratio of a pair of trips using the same set of sampled trip pairs in Figure 3(b). Comparing with the Figure 3(b), we see 
that the linear regression fit has a slope closer to 1, which means the two ratios are closer, and the $R^{2}$ is larger, which means the line fits better.

\subsection{Time Complexity Analysis}

Our approach has three steps: (1) mapping training trips into grids; (2) extracting neighbors of a given OD pair; and (3) estimating travel time based on the neighbors.

Step 1. To quickly retrieve the neighboring trips, we employ a raster partition of the city (e.g., 50 by $50 \mathrm{~m}$ grid in our experiment), and preparing $N$ training trips takes $O(N)$ time. This step can be preprocessed offline.

Step 2. Given a testing trip q, we find its corresponding origin gird in $O(1)$ time. Then, retrieving all the neighboring trips in the same grid would take $O(N / h)$ time if the trips are uniformly distributed, where $h$ is the total number of grids. In practice, however, the number of trips in each grid follows a long tail distribution. Therefore, the worst case time complexity of retrieving neighboring trips would be $O(\alpha \cdot N)$, where $\alpha \cdot N$ is the number of trips in the most dense grid $(\alpha=0.01$ in NYC taxi data).

Step 3. After retrieving the neighboring trips, the time complexity of calculating travel time of $\mathbf{q}$ is $O(|\mathcal{N}(\mathbf{q})|)$, where $|\mathcal{N}(\mathbf{q})|$ is the number of neighbors of trip $\mathbf{q}$. The speed references can be computed offline. The relative speed reference takes $O(N)$ time, and ARIMA model can be trained in $O\left(M^{2}\right)$ time, where $M$ is the number of time slots. During the online estimation, lookingup relative speed reference takes $O(1)$ time, whereas using ARIMA to predict the current speed reference takes $O((p+q) \cdot d)$ time, where $p, q$, and $d$ are the parameters learned during model training ( $p=2, q=0$, and $d=1$ in our experiment).

Therefore, the time complexity of online computation is $O(\alpha \cdot N)$ in the worst case. To serve a large amount of batch queries, we can use multithreading to further boost the estimation time.

\section{EXPERIMENT}

In this section, we present a comprehensive experimental study on two real datasets. All the experiments are conducted on a 8 -core $3.4 \mathrm{GHz}$ Intel Core i7 system with 16GB memory. We parallelize the computation, which will be discussed in Section 5.6 in detail.

\subsection{Dataset}

We conduct experiments on datasets from two different countries to show the generality of our approach.

5.1.1 NYC Taxi. A large-scale New York City taxi dataset has been made public online [29]. The dataset contains more than 160 million taxi trips per year. Each trip contains information about pick-up location and time, drop-off location and time, trip distance, fare amount, and so on. We use the subset of trips within the borough of Manhattan (the boundary is obtained from wikimapia.org) from 2013 to 2015, which has roughly over 127 million trips per year. On average, we have 349,410 trips per day.

Figure 9(a) shows the distribution of GPS points of the pick-up and drop-off locations. Figure 9(b) shows the number of trips per day over the year 2013. Figures 9(c) and 9(d) show the empirical CDF plot of the trip distance and trip time. About 56\% trips have trip time less than $10 \mathrm{~min}$, and about $99 \%$ trips have trip time less than 30min. The mean and median trip times are 636 (s) and 546 (s), respectively. The mean and median trip distance are 1.935 (mile) and 1.6 (mile), respectively. We noticed that most of the trips in our dataset are short; however, to the best of our knowledge the NYC taxi dataset is the largest publicly accessible dataset. 


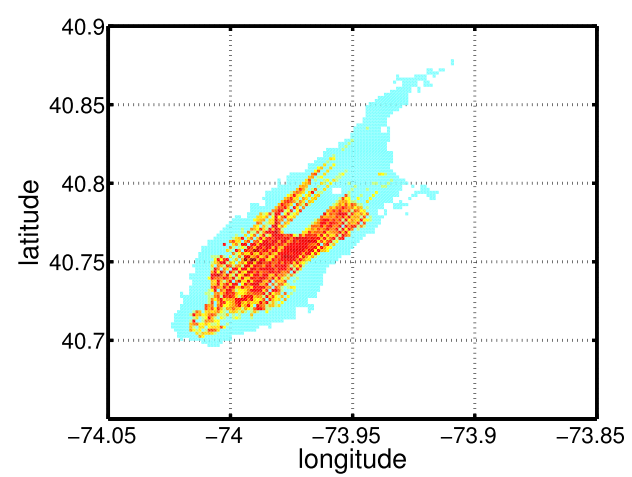

(a) Spatial distribution (red means dense location)

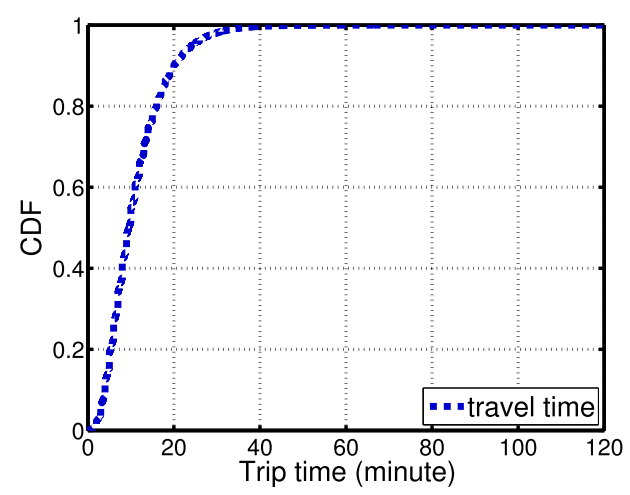

(c) Trip time

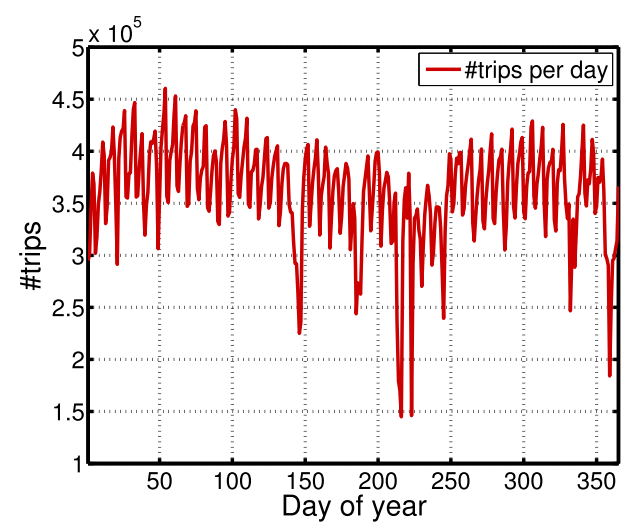

(b) Number of trips per day

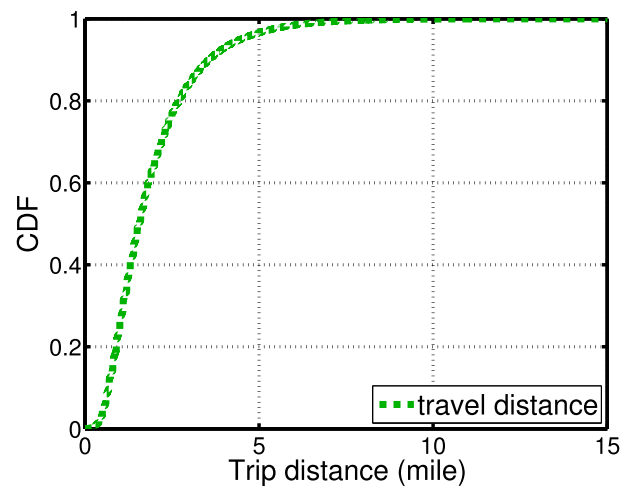

(d) Trip distance

Fig. 9. NYC data statistics. See text for explanations.

5.1.2 Shanghai Taxi. To compare with the existing route-based methods, we use the Shanghai taxi dataset with the trajectories of 2,600 taxis during two months in 2006 [25]. A GPS record has following fields: vehicle ID, speed, longitude, latitude, occupancy, and timestamp. In total, we have over 300 million GPS records. We extract the geographical information of Shanghai road networks from OpenStreetMap.

To retrieve taxi trips in this dataset, we rely on the occupancy bit of GPS records. This occupancy bit is 1 if there are passengers on board, and 0 otherwise. For each taxi, we define a trip as consecutive GPS records with occupancy equal to 1 . We get 5, 815, 470 trips after processing the raw data. The distribution of trip travel time is similar to that of NYC taxi data in Figure 9(c). Specifically, about half of the trips have travel time less than $10 \mathrm{~min}$.

\subsection{Evaluation Protocol}

Methods for evaluation. We systematically compare the following methods:

- Linear regression (LR). We train a linear regression model with travel time as a function of the L1 distance between pick-up and drop-off locations. This simple linear regression serves as a baseline for comparison.

- Neighbor average (AVG). This method simply takes the average of travel times of all neighboring trips as the estimation. 
- Temporally weighted neighbors (TEMP). This is our proposed method using temporal speed reference to assign weights on neighboring trips. We name the method using relative-time speed reference as TEMP rel (Section 4.1). And we call the method using ARIMA model to predict absolute temporal reference as TEMP abs (Section 4.2).

- Temporal speed reference by region $(T E M P+R)$. This is our improved method based on TEMP by considering the temporal reference for different region pairs (Section 4.3).

- Segment-based estimator (SEGMENT). This method estimates the travel time of each road segment individually, and then aggregate them to get the estimation of a complete trip (a baseline method used in Reference [27]).

- Subpath-based estimator (SUBPATH). One drawback of SEGMENT is that the transition time at intersections cannot be captured. Therefore, Wang et al. [27] propose to concatenate subpaths to estimate the target route, where each sub-path is consisted of multiple road segments. For each sub-path, SUBPATH estimates its travel time by searching all the trips that contain this sub-path.

- Online map service (BING and BAIDU). We also compare our methods with online map services. We use Bing Maps [16] for NYC taxi dataset and Baidu Maps [1] for Shanghai dataset. We use Bing Maps instead of Google Maps, because Bing Maps API allows query with current traffic for free whereas Google does not provide that. To consider traffic, we send queries to Bing Maps at the same time of the same day (in a weekly window) as the starting time of the testing trip. Due to national security concerns, the mapping of raw GPS data is restricted in China [18]. So we use Baidu Maps instead of Bing Maps for Shanghai taxi dataset.

Evaluation metrics. Similar to Reference [27], we use mean absolute error (MAE) and mean relative error (MRE) to evaluate the travel time estimation methods:

$$
M A E=\frac{\sum_{i}\left|y_{i}-\hat{y}_{i}\right|}{n}, M R E=\frac{\sum_{i}\left|y_{i}-\hat{y}_{i}\right|}{\sum_{i} y_{i}},
$$

where $\hat{y}_{i}$ is the travel time estimation of test trip $i$ and $y_{i}$ is the ground truth. Since there are anomalous trips, we also use the median absolute error (MedAE) and median relative error (MedRE) to evaluate the methods:

$$
\operatorname{MedAE}=\operatorname{median}\left(\left|y_{i}-\hat{y}_{i}\right|\right), \operatorname{MedRE}=\operatorname{median}\left(\frac{\left|y_{i}-\hat{y}_{i}\right|}{y_{i}}\right),
$$

where median returns the median value of a vector.

\subsection{Performance on NYC Data}

5.3.1 Overall Performance on NYC Data. In our experiment, we evaluate our methods independently within each of the three years. The trips from the first 11 months (i.e., January to November) are used as training and the ones in the last month (i.e., December) are used as testing. As for the BING method, due to the limited quota, we sample 260k trips in December as testing of each year. Bing Map API does not support travel time query of a past trip. Therefore, we assume that the traffic condition has strong weekly periodicity, and we use the same relative time within a week to query the Bing prediction for all the testing trips. The Bing predictions of all testing trips are queried in the third week of August 2017. The overall accuracy comparisons are shown in Table 1. Since NYC data only have endpoints of trips, we cannot compare our method with route-based methods. We will compare with these methods using Shanghai data in Section 5.4.

We first observe that our method is better than linear regression (LR) baseline. This is expected because LR is a simple baseline that does not consider the origin and destination locations. 
Table 1. Overall Performance on NYC Data

\begin{tabular}{|c|c|c|c|c|c|}
\hline Year & Method & MAE (s) & MRE & MedAE (s) & MedRE \\
\hline \multirow{6}{*}{2013} & LR & 194.60 & 0.2949 & 164.82 & 0.3017 \\
\hline & AVG & 178.46 & 0.2704 & 120.83 & 0.2345 \\
\hline & TEMP $_{\text {rel }}$ & 149.81 & 0.2270 & 97.36 & 0.1907 \\
\hline & TEMP $_{\text {abs }}$ & 143.31 & 0.2171 & 98.78 & 0.1890 \\
\hline & TEMP $_{\text {rel }}+\mathrm{R}$ & 143.72 & 0.2178 & 92.07 & 0.1805 \\
\hline & $\mathrm{TEMP}_{\mathrm{abs}}+\mathrm{R}$ & 142.33 & 0.2157 & 98.05 & 0.1874 \\
\hline \multirow{6}{*}{2014} & LR & 291.76 & 0.3552 & 224.574 & 0.3338 \\
\hline & $\overline{A V G}$ & 237.09 & 0.2887 & 142.17 & 0.2397 \\
\hline & TEMP $_{\text {rel }}$ & 204.43 & 0.2489 & 117.31 & 0.1993 \\
\hline & TEMP $_{\text {abs }}$ & 195.05 & 0.2375 & 118.90 & 0.1975 \\
\hline & TEMP $_{\text {rel }}+\mathrm{R}$ & 194.81 & 0.2371 & 110.179 & 0.1867 \\
\hline & $\mathrm{TEMP}_{\text {abs }}+\mathrm{R}$ & 192.69 & 0.2346 & 118.37 & 0.1956 \\
\hline \multirow{6}{*}{2015} & LR & 300.67 & 0.3567 & 227.87 & 0.3348 \\
\hline & AVG & 244.63 & 0.2902 & 147.10 & 0.2432 \\
\hline & TEMP $_{\text {rel }}$ & 207.64 & 0.2463 & 120.06 & 0.2000 \\
\hline & TEMP $_{\text {abs }}$ & 196.62 & 0.2332 & 116.78 & 0.1928 \\
\hline & $\mathrm{TEMP}_{\text {rel }}+\mathrm{R}$ & 196.54 & 0.2331 & 111.91 & 0.1859 \\
\hline & $\mathrm{TEMP}_{\mathrm{abs}}+\mathrm{R}$ & 196.61 & 0.2332 & 116.78 & 0.1928 \\
\hline \multicolumn{6}{|c|}{ Comparison with Bing Maps (260K testing trips per year) } \\
\hline \multirow{3}{*}{2013} & BING & 233.81 & 0.3642 & 153.00 & 0.3180 \\
\hline & BING(traffic) & 193.53 & 0.3015 & 140.00 & 0.2678 \\
\hline & $\mathrm{TEMP}_{\mathrm{abs}}+\mathrm{R}$ & 135.36 & 0.2108 & 94.94 & 0.1839 \\
\hline \multirow{3}{*}{2014} & BING & 343.69 & 0.4118 & 205.00 & 0.3615 \\
\hline & BING(traffic) & 240.41 & 0.2915 & 153.00 & 0.2517 \\
\hline & $\mathrm{TEMP}_{\mathrm{abs}}+\mathrm{R}$ & 192.75 & 0.2337 & 117.59 & 0.1947 \\
\hline \multirow{3}{*}{2015} & BING & 381.92 & 0.4410 & 231.00 & 0.3866 \\
\hline & BING(traffic) & 250.49 & 0.2892 & 152.00 & 0.2453 \\
\hline & $\mathrm{TEMP}_{\mathrm{abs}}+\mathrm{R}$ & 204.12 & 0.2357 & 119.93 & 0.1938 \\
\hline
\end{tabular}

Considering temporal variations of traffic condition improves the estimation performance. All the TEMP methods have significantly lower errors compared with AVG method. The improvement of TEMP ${ }_{a b s}$ over AVG is about 35-50s in MAE. In other words, the MAE is decreased by nearly $20 \%$ by considering the temporal factor. Furthermore, using the absolute speed reference (TEMP $\left.{ }_{a b s}\right)$ is better than using the relative (i.e., weekly) speed reference $\left(T_{E M P}\right.$ rel $)$. This is because the traffic condition does not strictly follow the weekly pattern, but has some irregular days such as holidays.

By adding the region factor, the performance is further improved. This means the traffic patterns between regions are actually different. However, we observe that the improvement of TEMP $\mathrm{abs}_{\mathrm{a}}+\mathrm{R}$ over TEMP $a$ bs is not as significant as the improvement of TEMP $\mathrm{rel}_{\text {rel }}+\mathrm{R}$ over TEMP rel. This is due to data sparsity when we compute the absolute time reference for each region pair. If two regions have little traffic flow, then the absolute time reference might not be accurate. In this case, using the relative time reference between regions make the data more dense and produces better results.

In Table 1 , the MAE of TEMP ${ }_{\text {abs }}+\mathrm{R}$ is significantly lower than BING by more than 100s. BING underestimates the travel times without considering traffic, where $81.37 \%$ testing trips are underestimated. When considering traffic, BING(traffic) is able to better predict the travel time. 


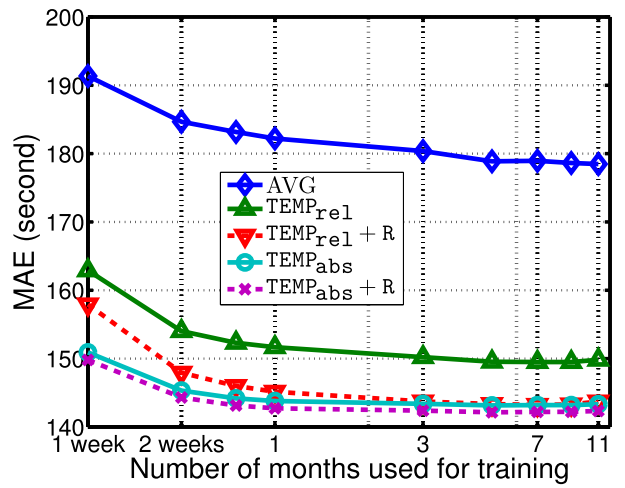

(a) MAE w.r.t. training set size

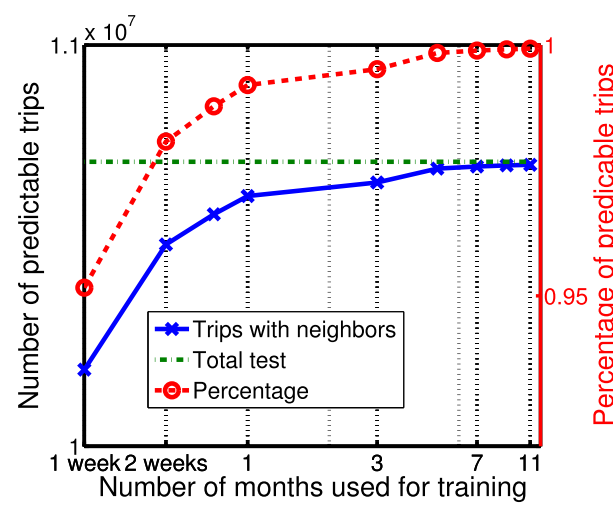

(b) Coverage of testing set

Fig. 10. Performance with regard to the training set size on 2013 data.

Notice that this result is different from our conference paper [24], where BING(traffic) has a higher prediction error than BING. The reason is that the previous Bing results are queried in October 2015. In the this article, we queried the Bing Map predictions for testing trips in the week of August 2017. There might be an improvement on the Bing Map service.

5.3.2 Performance w.r.t. the Size of Historical Data. We expect that, by using more historical data (i.e., training data), the estimation will be more accurate. To verify this, we study how performance changes w.r.t. the size of training data. We choose different time durations (from 1 week, 2 weeks, to 11 months) before December as the training data. Figure 10(a) shows the accuracy w.r.t. the size of the training data. The result meets with our expectation that MAE drops when using more historical data. But the gain of using more data is not obvious when using more than 1 month data. This indicates that using 1-month training data is enough to achieve a stable performance in our experimental setting. Such indication will save running time in real applications as we do not need to search for neighboring trips more than 1 month ago.

In addition, using more historical data, we are able to cover more testing trips. Because a testing trip should have at least one neighboring trip to be estimated. Figure 10(b) shows the coverage of testing data by using more historical data. With 1-week data, we can estimate $95.16 \%$ testing trips; with 1-month data, we can cover $99.20 \%$ testing trips.

5.3.3 Performance w.r.t. Trip Features. Next, we study the performance of all methods with respect to various trip features.

Performance w.r.t. trip time. In Figures 11(a) and 11(b), we plot the MAE and MRE with respect to the trip time. As expected, the longer-time trips have higher MAEs. It is interesting to observe that the MREs are high for both short-time trips and long-time trips. Because the shorttime trips (less than 500s) are more sensitive to dynamic conditions of the trips, such as traffic light. However, the long-time trips usually have less neighbors, which leads to higher MREs.

Performance w.r.t. trip distance. The MAE and MRE against trip distance are shown in Figures 11(c) and 11(d). Overall, we have consistent observations as Figures 11(a) and 11(b). However, we notice that the error of $\mathrm{TEMP}_{\text {rel }}+\mathrm{R}$ increases faster than other methods. $\mathrm{TEMP}_{\mathrm{rel}}+\mathrm{R}$ becomes even worse than AVG method when the trip distance is longer than 8 miles. This is because the longer-distance trips have fewer neighboring trips. However, we do not observe the same phenomenon in Figures 11(a) and 11(b). After further inspection, we find that long-distance trips have even less neighboring trips than long-time trips. The reason is that long-distance trips usually have 


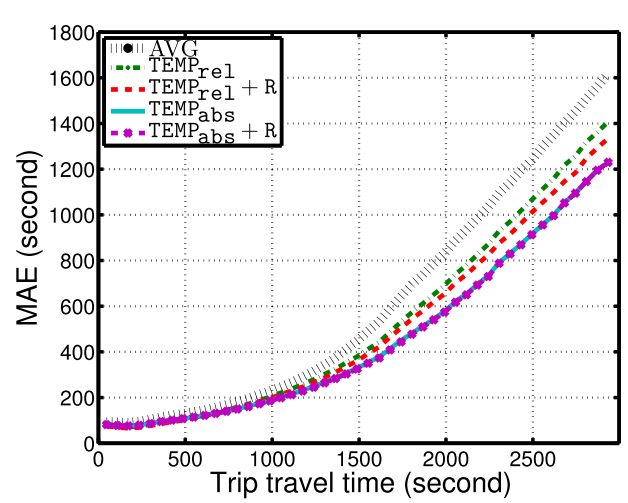

(a) MAE w.r.t. travel time

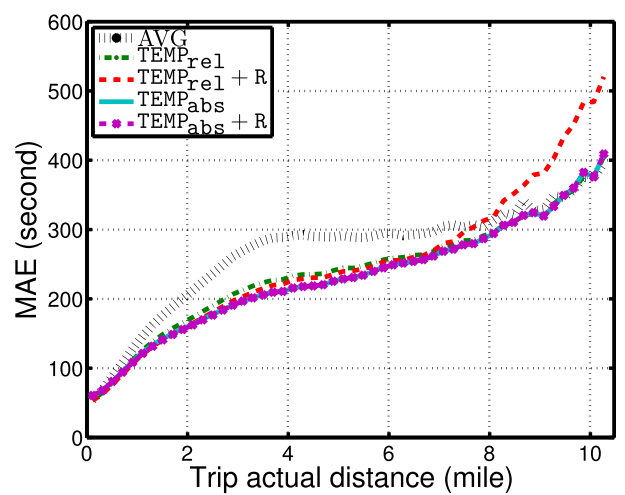

(c) MAE w.r.t. distance

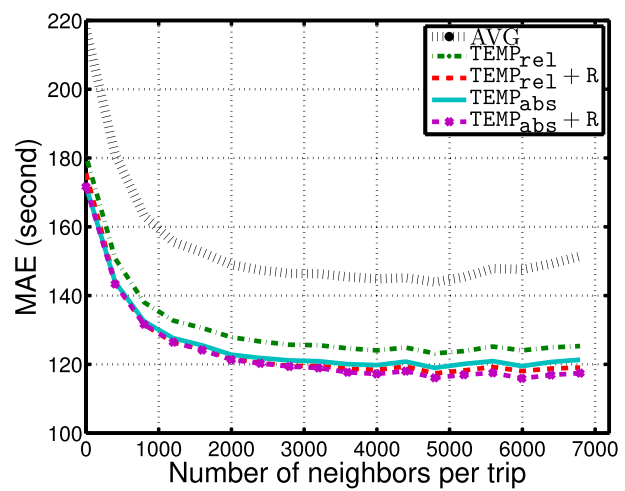

(e) MAE w.r.t. \# neighbors

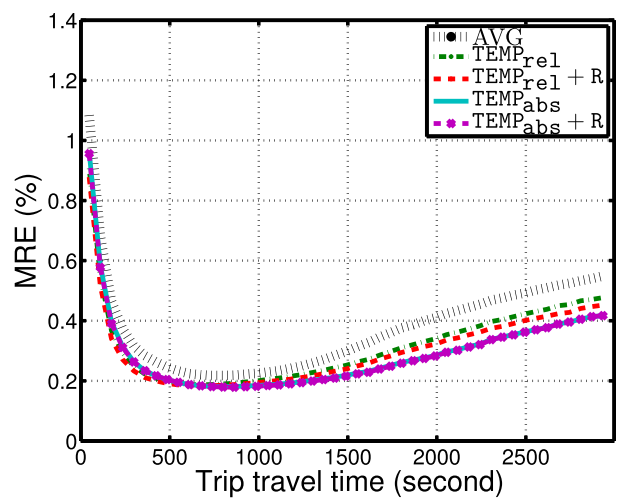

(b) MRE w.r.t. travel time

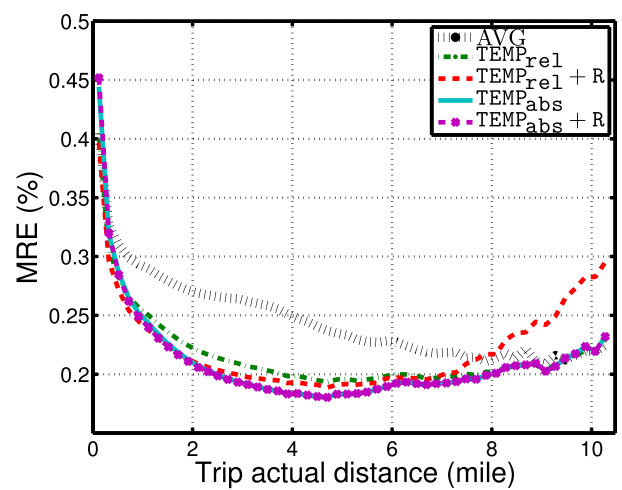

(d) MRE w.r.t. distance

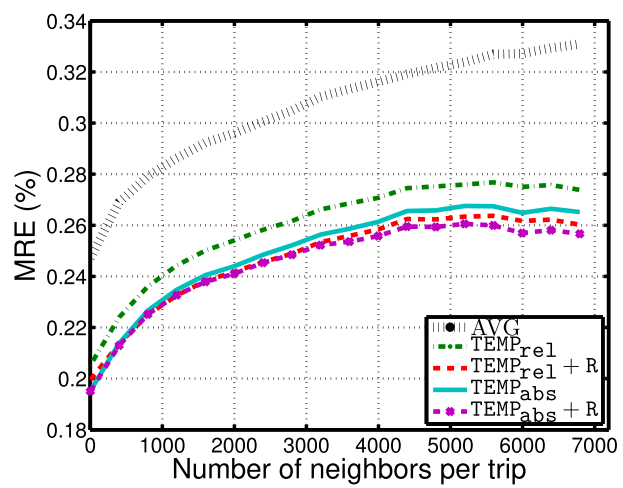

(f) MRE w.r.t. \# neighbors

Fig. 11. Performance with regard to the trip features.

long travel time; however, long-time trips may not have long trip distance (e.g., the long travel time may be due to traffic).

Performance w.r.t. number of neighboring trips. The error against number of neighbors per trip is shown in Figures 11(e) and 11(f). MAEs decrease for the trips with more neighbors. However, MREs increase as the number of neighbors increases. The reason is that the short trips 
Table 2. Overall Performance on Shanghai Taxi Data

\begin{tabular}{|l|c|c|c|c|}
\hline Method & MAE $(\mathrm{s})$ & MRE & MedAE $(\mathrm{s})$ & MedRE \\
\hline \hline LR & 130.710 & 0.6399 & 138.796 & 0.6173 \\
\hline BAIDU & 111.484 & 0.5451 & 73.001 & 0.5001 \\
\hline \hline SEGMENT & 119.833 & 0.5866 & 84.704 & 0.4947 \\
\hline SUBPATH & 113.566 & 0.5560 & 75.913 & 0.4820 \\
\hline \hline AVG & 94.202 & 0.4615 & 60.183 & 0.3739 \\
\hline TEMP $_{\text {rel }}$ & $\mathbf{9 2 . 4 2 8}$ & $\mathbf{0 . 4 5 2 7}$ & $\mathbf{5 5 . 3 1 7}$ & $\mathbf{0 . 3 6 7 8}$ \\
\hline
\end{tabular}

usually have more neighbors and short trips have higher relative errors, as shown in Figures 11(b) and $11(\mathrm{~d})$.

5.3.4 An Alternative Definition of Neighbors. We further study the performance by defining soft neighbors. We give neighboring trips spatial weights based on the sum of corresponding end-points' distance, and evaluate the performance on 500k trips. The MAE of TEMPabs $+R$ is $107.705 \mathrm{~s}$ on this testing set. Adding spatial weights on the neighbors degenerates the performance to 112.392s. It suggests that it is non trivial to assign weights on neighbors based on the distances of end-points. It will require more careful consideration of this factor.

\subsection{Performance on Shanghai Taxi Data}

In this section, we conduct experiments on Shanghai taxi data. This dataset provides the trajectories of the taxi trips, so we can compare our method with SEGMENT and SUBPATH methods. Both SEGMENT and SUBPATH methods use the travel time on individual road segments or subpaths to estimate the travel time for a query trip. Due to data sparsity, we cannot obtain travel time for every road segments. Reference [27] proposes a tensor decomposition method to estimate the missing values. In our experiment, to avoid the missing value issue, we only select the testing trips that have values on every segments of the trip. However, by doing such selection, the testing trips are biased towards shorter trips. Because the shorter the trip is, the less likely the trip has missing values on the segment. To alleviate this bias issue, we further sampled the biased trip dataset to make the travel time distribution similar to the distribution of the original whole dataset. We randomly sample 2,138 trips in total as testing, and the rest are used as training. We use the same training set and testing set for different methods.

5.4.1 Overall Performance on Shanghai Taxi Data. The comparison among different methods is shown in Table 2. Our neighbor-based method significantly outperform other methods. The simple method AVG is $17 \mathrm{~s}$ better than BAIDU in terms of MAE. By considering temporal factors, our method TEMP $_{\text {rel }}$ further outperforms AVG method. Here, we do not report the results of TEMP ${ }_{\text {abs }}$, because the two-month Shanghai data have several days' trip missing, but the ARIMA model requires complete time series. In addition, SUBPATH method outperforms SEGMENT method, which is consistent with previous work [27]. SEGMENT simply adds travel time of individual segments, whereas SUBPATH considers the transition time between segments by concatenating subpaths. However, SUBPATH is still 21s worse than our method TEMP rel in terms of MAE. Such result is also expected. Because our method can be considered as a special case of SUBPATH method, where we use the whole paths from the training data to estimate the travel time for a testing trip. If we have enough number of whole paths, then it is better to use the whole paths instead of subpaths.

Since the Shanghai taxi data are much more sparse than NYC data, we do not show the results of $\mathrm{TEMP}_{\text {rel }}+\mathrm{R}$ and $\mathrm{TEMP}_{\mathrm{abs}}+\mathrm{R}$ on this dataset. Note that the main goal of this experiment is to 


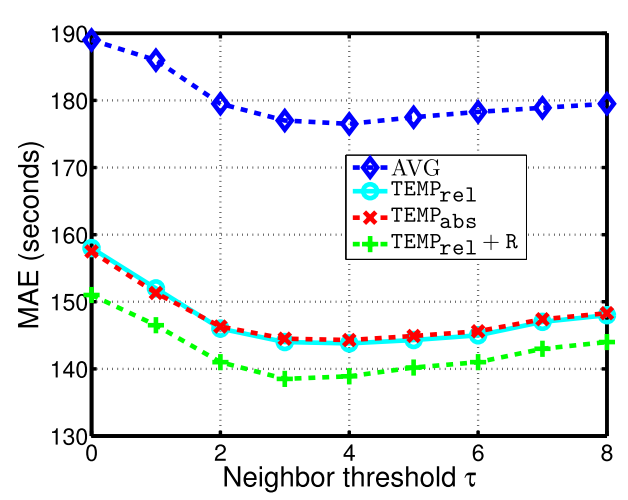

(a) Performance

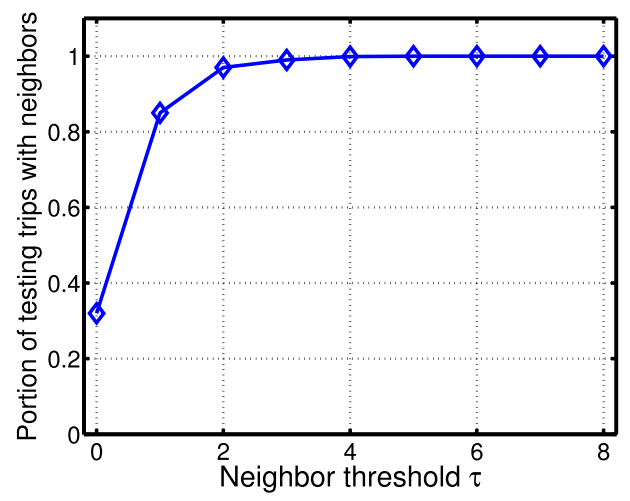

(b) Coverage

Fig. 12. Estimation error and testing coverage w.r.t. neighbor threshold $\tau$.

show that TEMP rel outperforms the existing methods. With more data, our other approaches could potentially outperform the TEMPrel as well as shown in NYC data.

5.4.2 Applicability of Segment-based Method and Neighbor-based Method. Segment-based method requires every individual road segment of the testing trip has at least one historical data point to estimate the travel time. However, our neighbor-based method requires a testing trip has at least one neighboring trip. Among 435,887 trips in Shanghai dataset, only 54,530 trips have values on every road segment, but 217,585 trips have at least one neighbor. Therefore, in this experimental setting, our method not only outperforms segment-based method in terms of accuracy, it is also more applicable to answer more queries (49.9\%) compared with segment-based method $(12.5 \%)$.

\subsection{Parameter Sensitivity}

In this section, we study the parameter sensitivity of our proposed method. There is only one parameter in our proposed method, the distance threshold $\tau$ to define the neighbors. We use the first 11 months of 2013 from NYC dataset to study the performance w.r.t. parameter $\tau$. For computational efficiency, we partition the map into small grids of 50 by $50 \mathrm{~m}$. The distance in Equation (1) is now defined as the L1 distance between two grids. For example, if $p_{1}$ is a neighboring trip of $q$ for $\tau=0$, it means that the pick-up (and drop-off) location of $p_{1}$ is in the same grid as the pick-up (and drop-off) location of $q$. If $\tau=1$, then a neighboring trip has endpoints in the same or adjacent grids.

A larger $\tau$ will retrieve more neighboring trips for a testing trip and thus could give an estimation with a higher confidence. However, a larger $\tau$ also implies that the neighboring trips are less similar to the testing trip, which could introduce prediction errors. Therefore, it is crucial to identify the optimal $\tau$ that balance estimation confidence and neighbors' similarity. In Figure 12(a), we plot the empirical relationship between the MAE and $\tau$. We can see that MAE is the lowest when $\tau$ is in the range of $[3,6]$, striking a good balance between confidence and similarity. It is worthy to mention that our method is not sensitive to the different selection of $\tau$.

We also want to point out that we need to have at least one neighboring trip to give an estimation of a testing trip. Obviously, the larger $\tau$ is, the more testing trips we can cover. Figure 12(b) plots the percentage of testing trips with at least one neighbor. The figure shows that if $\tau=1$, there are only $32 \%$ trips are predictable. When $\tau=3,99 \%$ trips are predictable. Considering the both aspects, we use $\tau=3$ in our experiments, which considers two trips are neighboring if their starting and ending 
Table 3. Running Time Comparison

\begin{tabular}{|c|c|c|c|c|}
\hline Method & Process & No. trips & Time & Time/trip \\
\hline \multirow{3}{*}{$\begin{array}{c}\text { Our } \\
\text { method }\end{array}$} & Prep. training trip & $11 \mathrm{M}$ & $42 \mathrm{~s}$ & $0.004 \mathrm{~ms}$ \\
\cline { 2 - 5 } & Learn ARIMA & $11 \mathrm{M}$ & $147 \mathrm{~s}$ & $0.013 \mathrm{~ms}$ \\
\cline { 2 - 5 } & Find neighbor & $100 \mathrm{~K}$ & $24 \mathrm{~s}$ & $0.24 \mathrm{~ms}$ \\
\cline { 2 - 5 } & Estimate & $10.5 \mathrm{M}$ & $229 \mathrm{~s}$ & $0.021 \mathrm{~ms}$ \\
\hline \multirow{2}{*}{ SUBPATH } & Prep. training trip & $4.35 \mathrm{M}$ & $547 \mathrm{~s}$ & $0.126 \mathrm{~ms}$ \\
\cline { 2 - 5 } & Estimate & $54 \mathrm{~K}$ & $2,297 \mathrm{~s}$ & $42 \mathrm{~ms}$ \\
\hline
\end{tabular}

points are within $150 \mathrm{~m}$. Our method can also adapt to different cities by controlling neighborhood parameter $\tau$.

\subsection{Running Time}

Table 3 presents the running time comparison between TEMP ${ }_{a b s}+\mathrm{R}$ and SUBPATH. We use a multithreading implementation of our method, since it is easy to parallel. We profile the running time of TEMP ${ }_{\text {abs }}+\mathrm{R}$ on last two months' trips in NYC dataset, and SUBPATH on whole Shanghai dataset. Note that NYC dataset is bigger than Shanghai dataset and TEMP ${ }_{a b s}+\mathrm{R}$ is the most computationally expensive method and also the most accurate method among our proposed methods. The pre-processing time for $\mathrm{TEMP}_{\mathrm{abs}}+\mathrm{R}$ consists of mapping trips to grids, calculating the speed reference, and learning the ARIMA parameters. The preparation time for SUBPATH includes indexing trajectory by road segments.

In TEMP abs $+\mathrm{R}$, both training trip preparation and learning ARIMA can be done off-line or updated every hour with the new data. The online query part includes querying neighboring trips and estimation using neighbors. The neighbor finding is the most time-consuming part. We get $1.09 \mathrm{~ms} /$ trip if using one thread and $0.24 \mathrm{~ms} /$ trip if using eight threads. The estimation part only takes $0.02 \mathrm{~ms}$ with eight threads. So the total online query time for our method is $1.505 \mathrm{~ms} /$ trip on average and it will be even faster (i.e., $0.26 \mathrm{~ms} /$ trip) if using eight threads. The estimation of SUBPATH includes finding optimal concatenation of segments, which has $O\left(n^{2} \cdot m\right)$ time complexity, where $n$ is the number of road segments and $m$ is number of trips going through each segment. It takes $42 \mathrm{~ms}$ on average to estimate a testing trip. Our neighbor-based method is more efficient in answering travel time queries, because we avoid computing the route and estimating the time for the subpaths.

\section{CONCLUSION}

This article demonstrates that one can use large-scale trip data to estimate the travel time between an origin and a destination in a very efficient and effective way. Our proposed method retrieves all the neighboring historical trips with similar origin and estimation locations and estimates the travel time using those neighboring trips. We further improve our method by considering the traffic conditions w.r.t. different temporal granularity and spatial dynamics. We conduct experiments on two large-scale real-world datasets and show that our method can greatly outperform the stateof-the-art methods and online map services.

In our method, the spatial region is an important factor that greatly improves the estimation accuracy. However, for the purpose of simplicity, we directly employ the administrative boundary to partition the city into regions. One could improve this baseline partitioning by learning a better partitioning from the taxi data, because the goal of region partition should be finding neighborhoods within which the temporal traffic pattern is similar. Deriving those neighborhoods with similar traffic patterns is non-trivial and worth further investigation. 


\section{ACKNOWLEDGMENTS}

The authors thank the anonymous referees for their valuable comments and helpful suggestions.

\section{REFERENCES}

[1] Baidu 2016. Baidu Map. Retrieved from http://map.baidu.com.

[2] Paolo Cintia, Roberto Trasarti, Livia Almada Cruz, Camila F. Costa, and Jose Antônio F. de Macedo. 2013. A gravity model for speed estimation over road network. In Proceedings of the IEEE International Conference on Mobile Data Management (MDM'13), Vol. 2. IEEE, 136-141. DOI : https://doi.org/10.1109/MDM.2013.83

[3] Corrado De Fabritiis, Roberto Ragona, and Gaetano Valenti. 2008. Traffic estimation and prediction based on real time floating car data. In Proceedings of the IEEE International Conference on Intelligent Transportation Systems. 197-203.

[4] Brian C. Dean. 1999. Continuous-time Dynamic Shortest Path Algorithms. Ph.D. Dissertation. Massachusetts Institute of Technology.

[5] Hector Gonzalez, Jiawei Han, Xiaolei Li, Margaret Myslinska, and John Paul Sondag. 2007. Adaptive fastest path computation on a road network: A traffic mining approach. In Proceedings of the International Conference on Very Large Data Bases. 794-805.

[6] Ryan Herring, Aude Hofleitner, Saurabh Amin, T. Nasr, A. Khalek, Pieter Abbeel, and Alexandre Bayen. 2010. Using mobile phones to forecast arterial traffic through statistical learning. In Proceedings of the 89th Transportation Research Board Annual Meeting. 1-22.

[7] Aude Hofleitner and Alexandre Bayen. 2011. Optimal decomposition of travel times measured by probe vehicles using a statistical traffic flow model. In Proceedings of the IEEE International Conference on Intelligent Transportation Systems. $815-821$.

[8] Aude Hofleitner, Ryan Herring, Pieter Abbeel, and Alexandre Bayen. 2012. Learning the dynamics of arterial traffic from probe data using a dynamic Bayesian network. IEEE Trans. Intell. Transport. Syst. 13, 4 (2012), 1679-1693.

[9] Timothy Hunter, Ryan Herring, Pieter Abbeel, and Alexandre Bayen. 2009. Path and travel time inference from GPS probe vehicle data. NIPS Analyz. Netw. Learn. Graphs 12, 1 (2009).

[10] Rob J. Hyndman and George Athanasopoulos. 2014. Forecasting: Principles and Practice. OTexts.

[11] Erik Jenelius and Haris N. Koutsopoulos. 2013. Travel time estimation for urban road networks using low frequency probe vehicle data. Transport. Res. Part B: Methodol. 53 (2013), 64-81.

[12] Zhanfeng Jia, Chao Chen, Ben Coifman, and Pravin Varaiya. 2001. The PeMS algorithms for accurate, real-time estimates of g-factors and speeds from single-loop detectors. In Proceedinsg of the IEEE International Conference on Intelligent Transportation Systems. 536-541.

[13] Evangelos Kanoulas, Yang Du, Tian Xia, and Donghui Zhang. 2006. Finding fastest paths on a road network with speed patterns. In Proceedings of the IEEE International Conference on Data Engineering. IEEE, 10-10.

[14] Feifei Li, Dihan Cheng, Marios Hadjieleftheriou, George Kollios, and Shang-Hua Teng. 2005. On trip planning queries in spatial databases. In Advances in Spatial and Temporal Databases. Springer, 273-290.

[15] $\mathrm{Mu} \mathrm{Li}, \mathrm{Amr}$ Ahmed, and Alexander J. Smola. 2015. Inferring movement trajectories from GPS snippets. In Proceedings of the ACM International Conference on Web Search and Data Mining (WSDM'15). 325-334.

[16] Microsoft Bing 2017. Bing Maps. Retrieved from https://www.bing.com/maps/.

[17] Enda Murphy and James E. Killen. 2011. Commuting economy: An alternative approach for assessing regional commuting efficiency. Urban studies 48, 6 (2011), 1255-1272.

[18] National Administration of Surveying, Mapping, and Geoinformation of China. 2016. Surveying and Mapping Law of the People's Republic of China. Chapter II, Article 9, 10.

[19] Michael A. Niedzielski. 2006. A spatially disaggregated approach to commuting efficiency. Urban Studies 43, 13 (2006), 2485-2502.

[20] Karl F. Petty, Peter Bickel, Michael Ostland, John Rice, Frederic Schoenberg, Jiming Jiang, and Ya'acov Ritov. 1998. Accurate estimation of travel times from single-loop detectors. Transport. Res. Part A: Policy Practice 32, 1 (1998), $1-17$.

[21] Mahmood Rahmani, Erik Jenelius, and Haris N. Koutsopoulos. 2013. Route travel time estimation using low-frequency floating car data. Proceedings of the IEEE International Conference on Intelligent Transportation Systems.

[22] John Rice and Erik Van Zwet. 2004. A simple and effective method for predicting travel times on freeways. IEEE Trans. Intell. Transport. Syst. 5, 3 (2004), 200-207.

[23] Martin Treiber and Arne Kesting. 2013. Traffic flow dynamics. Traffic Flow Dynamics: Data, Models and Simulation, Springer-Verlag, Berlin (2013).

[24] Hongjian Wang, Yu-Hsuan Kuo, Daniel Kifer, and Zhenhui Li. 2016. A simple baseline for travel time estimation using large-scale trip data. In Proceedings of the 24th SIGSPATIAL International Conference on Advances in Geographic Information Systems (GIS'16). ACM, New York, NY. 
[25] Hongjian Wang, Yanmin Zhu, and Qian Zhang. 2013. Compressive sensing-based monitoring with vehicular networks. In Proceedings IEEE International Conference on Computer Communications (INFOCOM'13). 2823-2831. DOI : https://doi.org/10.1109/INFCOM.2013.6567092

[26] Yibing Wang and Markos Papageorgiou. 2005. Real-time freeway traffic state estimation based on extended Kalman filter: A general approach. Transport. Res. Part B: Methodol. 39, 2 (2005), 141-167.

[27] Yilun Wang, Yu Zheng, and Yexiang Xue. 2014. Travel time estimation of a path using sparse trajectories. In Proceedings of the 20th ACM SIGKDD International Conference on Knowledge Discovery and Data Mining. ACM, 25-34.

[28] Bradford S. Westgate, Dawn B. Woodard, David S. Matteson, and Shane G. Henderson. 2013. Travel time estimation for ambulances using Bayesian data augmentation. Ann. Appl. Stat. 7, 2 (2013), 1139-1161.

[29] Chris Whong. 2014. Foiling NYC's Taxi Trip Data. Retrieved from http://chriswhong.com/open-data/foil_nyc_taxi/.

[30] Daniel B. Work, O.-P. Tossavainen, Sébastien Blandin, Alexandre M. Bayen, Toch Iwuchukwu, and Ken Tracton. 2008. An ensemble Kalman filtering approach to highway traffic estimation using GPS enabled mobile devices. In Proceedings of the IEEE Conference on Decision and Control. 5062-5068.

[31] Jing Yuan, Yu Zheng, Chengyang Zhang, Wenlei Xie, Xing Xie, Guangzhong Sun, and Yan Huang. 2010. T-drive: Driving directions based on taxi trajectories. In Proceedings of the ACM International Conference on Advances in Geographic Information Systems (ACM SIGSPATIAL'10). 99-108.

Received November 2016; revised August 2017; accepted October 2018 